\title{
Improving signal detection accuracy at FC of a CRN using machine learning and fuzzy rules
}

\author{
Md Abul Kalam Azad, Anup Majumder, Jugal Krishna Das, Md Imdadul Islam \\ Depatment of Computer Science and Engineering, Jahangirnagar University, Dhaka, Bangladesh
}

\begin{tabular}{|c|c|}
\hline Article Info & ABSTRACT \\
\hline Article history: & The performance of a cognitive radio network (CRN) mainly depends on the \\
\hline Received Jun 19, 2020 & faithful signal detection at fusion center (FC). In this paper, the concept of \\
\hline Revised Aug 11, 2020 & weighted fuzzy rule in Iris data classification, as well as, four machine \\
\hline Accepted Aug 30, 2020 & clustering (FCMC), support vector machine (SVM) and convolutional neural \\
\hline & $\begin{array}{l}\text { network }(\mathrm{CNN}) \text { are applied in signal detection at FC taking signal-to- } \\
\text { interference plus noise ratio of secondary users as parameter. The weighted }\end{array}$ \\
\hline Keywords: & fuzzy rule gave the detection accuracy of $86.6 \%$, which resembles the energy \\
\hline Co-operative CRN & detection model of majority rule of FC; however, CNN gave an accuracy of \\
\hline Data classification & $91.3 \%$ at the expense of more decision time. The FIS, FCMC and SVM gave \\
\hline Entropy & some intermediate results; however, the combined method gave the best \\
\hline Fusion center & result compared to that of any individual technique. \\
\hline
\end{tabular}

This is an open access article under the CC BY-SA license.

Fuzzy system

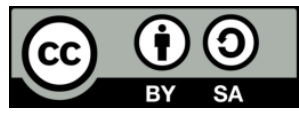

\section{Corresponding Author:}

\author{
Anup Majumder \\ Depatment of Computer Science and Engineering \\ Jahangirnagar University \\ Dhaka-1342, Bangladesh \\ Email: anupmajumder@juniv.edu
}

\section{INTRODUCTION}

In Cognitive Radio Network (CRN), there exists two types of users such as Primary User (PU) called licensed user and Secondary User (SU) called unlicensed user. A PU can access a traffic channel of the network when the channel is free; however, a SU is an opportunist user who can access a channel when the channel is not occupied by any PU. Moreover, a SU in service has to release the channel when it is claimed by a PU. Therefore, the detection accuracy of the presence of a PU is a key factor to avoid any misdetection and false alarm. Hence, the concept of co-operative CRN comes forth where the received signals of several SUs are combined at a Fusion Center (FC) to expedite the detection accuracy,

In contemporary works, Fuzzy logic and various machine learning techniques are used at a FC to improve the detection accuracy. The weighted Fuzzy rule or Fuzzy system is widely used in data classification problem of combined Membership Functions (MF) of input variables. It is used to classify Iris data where the weight of an input variable is determined from the range of a variable and its non-overlapping parts [1]. Since accuracy depends on labels, authors found the classification accuracy of $96.7 \%$ under 11 labels. The Fuzzy rule-based classification of coronary artery disease data is analyzed in [2]; where trapezoidal MFs are used as input variables. It is found that classification accuracy is varied on weighting rules with a maximum of $92.8 \%$ and a minimum of $71.8 \%$. A simulation work is done with relayed link communication to generate input data instead of importing them from a database. Seven different methods and Fuzzy $c$-Means Clustering [FCMC] are applied in magnetic resonance brain image classification problem and found a moderate performance [3]. A similar algorithm is applied for the classification of farms 
according to their financial health [4]. An image segmentation method based on Fuzzy clustering with cellular automata and feature weighting is proposed in [5]. A comparison is made among the proposed method, FCMC, K-means and Kernel FCMC.

Application of Support Vector Machine (SVM) for the classification of hand movement intentions is examined in [6]; where authors classified upto 52 hand movement intentions based on electromyography signals. It is shown that SVM based system gives a better result than that of least square twin SVM based system [7]. Four kernels named linear, polynomial, radial basis and sigmoid basis kernel are used to classify biomedical data among various dieases groups [8]. Author found the mean accuracy of 78.44\%, 62.8\%, $65.9 \%$ and $63.5 \%$ for linear, polynomial, radial basis and sigmoid basis kernel, respectively. Still, a research gap of SVM regarding its application in CRN is evident.

The application of SVM in spectrum sensing of CRN is found in [9]. The received signal is inputted to a tunable band pass filter and the output becomes a digital signal, which is then applied to an SVM. The performance of energy detection is compared with SVM on the plane of probability of false alarm and detection. A similar analysis is found in [10]. Two hypothesis model of CRN is used to determine the covariance matrix of received signal in [11]. Therefore, $N$ Eigen values determined from co-variance matrix are applied to kernel based SVM. The profile of probability of false alarm and misdetection against Signal-toNoise Ratio (SNR) are shown taking the number of antenna elements and input data as parameters. Instead of $\mathrm{N}$-dimensional energy vector, a low-dimensional probability vector is derived from multivariate Gaussian distribution function in [12], which is applied to SVM based classification. The authors claim that the probability vector of linear SVM has a better detection accuracy than the energy vector of previous work; however, $K$-means clustering performs slightly worse. A similar analysis and graphical result are also found in [13]. Still, we can use three level hypothesis with weighted Fuzzy rule or deep learning to observe the detection accuracy and processing time.

In [14], a 3-Dimensional Convolutional Neural Network (3-DCNN) is applied to age estimation in magnetic resonance imaging of human brain. The work makes a comparison with Principal Component Analysis, local features and 2-DCNN, and shows that 3-DCNN gives the best accuracy. The concept of CNN is also applied to sound classification over spectrograms [15]. The network is trained with a dataset consists of 6,776 spectrograms of different sounds, and the experiment gives an accuracy of $95 \%$ on training data set and an accuracy of $85 \%$ on test data set.

The concept of Deep Q-Network (DQN) to evaluate the capacity of a network is found in [16]; where SNR and Shannon formula are taken as input parameters, and data packets are divided in equal time slot keeping PU and SU synchronized. The variation of system capacity against time slot is plotted both for learning case i.e., DQN and for without learning case; where DQN gives a better result. However, the work ignores the fading model, as well as, the spectrum sensing model. The concept of FC is not considered either. The performance of $\mathrm{CRN}$ is determined at FC using the idea of co-operative spectrum sensing in OFDM system [17]. The probability of false alarm and detection is plotted against SNR for 16-QAM under Additive White Gaussioan Noise (AWGN). The CNN is applied to spectrum sensing and the impact of size and number of convolution layer, pooling layer and fully connected layer on time complexity are also analyzed. Still, we have the scope of using CNN under different fading environment to get a more realistic scenario for CRN.

In this paper, four popular machine learning methods i.e., FIS, FCMC, SVM and CNN along with Fuzzy weighted rule are applied in detecting the presence of PUs at FC; where the FC takes 16-QAM signal under AWGN and Rayleigh fading channel. Two conventional hypothesis models for signal detection are used in each method and finally, the accuracy levels of five methods are combined using Entropy.

The rest of the paper is organized as follows: Section 2 gives some basic theory of machine learning techniques to recognize the signal at FC, Section 3 deals with results based on the analysis of Section 2 and finally, Section 4 concludes entire analysis.

\section{THEORY OF DATA CLASSIFICATION}

In this section, we consider the basic theory of five data classification techniques: Fuzzy weighted rule, FIS, FCMC, SVM and CNN.

\subsection{Fuzzy weighted rule}

Here, Fuzzy weighted rule is explained with the help of two numerical examples. First of all, we take simulation data under two categories called $H_{0}$ and $H_{1}$ as shown in Table 1. For each category, four input parameters such as $\mathrm{SU}_{1}, \mathrm{SU}_{2}, \mathrm{SU}_{3}$ and $\mathrm{SU}_{4}$, and their corresponding output are shown explicitly in Table 2. In this paper, we use Fuzzy rules with five Membership Functions (MFs) as shown in Figure 1. 
Table 1. Input parameters and output type of the SU data from simulation

\begin{tabular}{llllllllll}
\hline \multicolumn{4}{c}{ Hypothesis $H_{0}$} & \multicolumn{6}{c}{ Hypothesis $H_{1}$} \\
$S U_{I}$ & $S U_{2}$ & $S U_{3}$ & $S U_{4}$ & Output & $S U_{1}$ & $S U_{2}$ & $S U_{3}$ & $S U_{4}$ & Output \\
\hline 0.064 & 1.688 & 0.824 & 0.314 & 1 & 2.820 & 2.151 & 3.224 & 5.221 & 2 \\
0.889 & 0.664 & 1.152 & 1.902 & 1 & 3.653 & 2.412 & 1.356 & 4.122 & 2 \\
0.553 & 0.079 & 0.221 & 0.981 & 1 & 4.312 & 3.443 & 3.089 & 1.209 & 2 \\
0.763 & 1.306 & 1.514 & 0.320 & 1 & 3.438 & 1.121 & 2.667 & 2.072 & 2 \\
0.453 & 0.919 & 0.231 & 1.331 & 1 & 2.494 & 3.411 & 4.108 & 3.109 & 2 \\
\hline
\end{tabular}

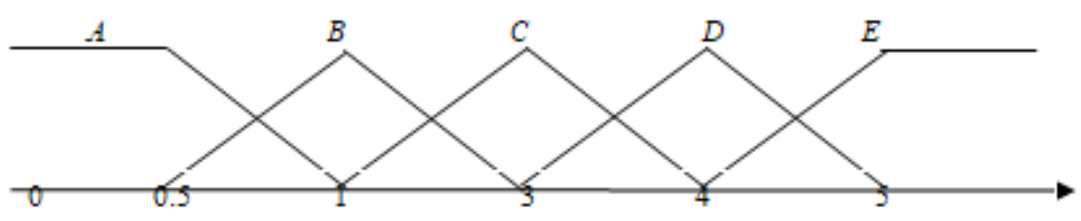

Figure 1. The MFs of the fyzzy system

Table 2. Input parameters and output type simulation data

\begin{tabular}{cccccccccc}
\hline \multicolumn{1}{c}{ Hypothesis $H_{0}$} & \multicolumn{6}{c}{ Hypothesis $H_{1}$} \\
$\mathrm{SU}_{1}$ & $\mathrm{SU}_{2}$ & $\mathrm{SU}_{3}$ & $\mathrm{SU}_{4}$ & Output & $\mathrm{SU}_{1}$ & $\mathrm{SU}_{2}$ & $\mathrm{SU}_{3}$ & $\mathrm{SU}_{4}$ & Output \\
\hline$A$ & $B$ & $B$ & $A$ & 1 & $C$ & $C$ & $C$ & $E$ & 2 \\
$B$ & $A$ & $B$ & $B$ & 1 & $D$ & $C$ & $B$ & $D$ & 2 \\
$A$ & $A$ & $A$ & $B$ & 1 & $D$ & $C$ & $C$ & $B$ & 2 \\
$B$ & $B$ & $B$ & $A$ & 1 & $C$ & $B$ & $C$ & $C$ & 2 \\
$A$ & $B$ & $A$ & $B$ & 1 & $C$ & $C$ & $D$ & $C$ & 2 \\
\hline
\end{tabular}

Now, the rule for $H_{0}$ is $R_{0}=\left((\{A, B\},\{A, B\},\{A, B\},\{A, B\}), H_{0}\right)$ and the rule for $H_{1}$ is $R_{1}=((\{C$, $\left.D\},\{B, C\},\{B, C, D\},\{C, B, D, E\}), H_{1}\right)$. We will explain the Fuzzy weighted rule through data validation techniques in a different way, specially using line diagrams and numerical examples.

\subsubsection{Numerical example-1}

Show that $\left(\mathrm{SU}_{1}, \mathrm{SU}_{2}, \mathrm{SU}_{3}, \mathrm{SU}_{4}\right) \equiv(0.72,0.83,1.71,0.134)$ belongs to output $H_{0}$.

From the membership function of SU signal, we get $(0.72,0.83,1.71,0.134) \leftrightarrow(\{A\},\{B\},\{B\}$, $\{A\})$. Considering the sets of rule $R_{0}, A \varepsilon\{A, B\}, B \in\{A, B\}, B \in\{A, B\}$, and $A \in\{A, B\}$. Therefore, $(0.72$, $0.83,1.71,0.134)$ belongs to the class $H_{0}$.

Using the theoretical analysis of $[1,2]$, we determine the Fuzzy weight factors. From the input of Table 1, the range of $\mathrm{SU}_{1}$ for output $H_{0}$ is 0.064 to 0.889 and for output $H_{1}$ is 2.494 to 4.312 as shown in Figure 2(a). For the convenience of analysis, the range of input data can be shown by line diagram as follows.

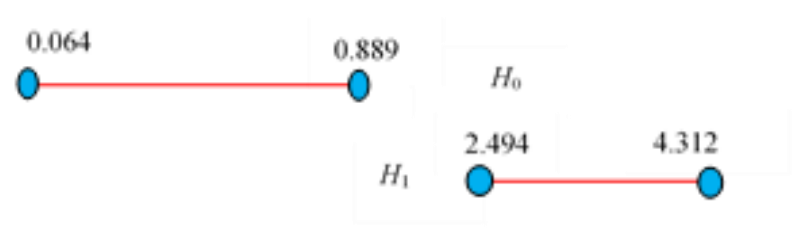

(a) Range of $\mathrm{SU}_{1}$

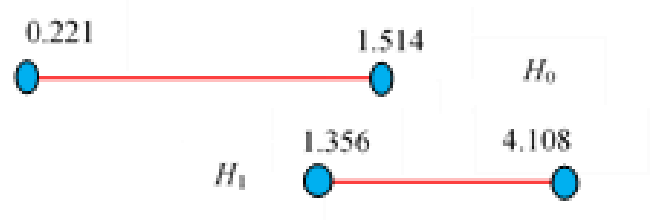

(c) Range of $\mathrm{SU}_{3}$

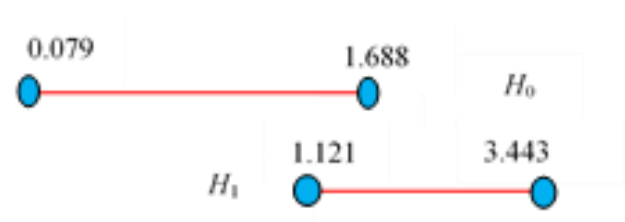

(b) Range of SU2

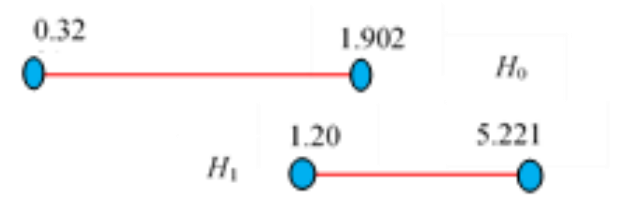

(d) Range of $\mathrm{SU}_{4}$

Figure 2. Range of input parameters of Table 1 
For input parameter of $\mathrm{SU}_{1}$, the line diagram becomes Figure 2(a). There is no overlapping part; therefore, the entire range $S$ and non-overlapping part, $S_{n}$ will be the same. Now, $S=(0.064-4.312)=S_{n}=$ 4.248; therefore, the ratio becomes, $V_{1}=4.248 / 4.248=1$. For $\mathrm{SU}_{2}$ of Figure $2(\mathrm{~b})$, the sum of nonoverlapping part, $S_{n}=(1.121-0.079)+(3.443-1.688)=2.797$. The entire range is $S=(3.443-0.079)=3.364$. Then the ratio becomes, $V_{\mathrm{i}}=\mathrm{S}_{\mathrm{n}} / \mathrm{S} \Rightarrow V_{2}=2.797 / 3.364=0.831$. With similar calculations of Figure 2(c) and Figure 2(d), we get $V_{3}=3.729 / 3.887=0.96$ for $\mathrm{SU}_{3}$, and $V_{4}=4.208 / 4.901=0.85$ for $\mathrm{SU}_{4}$, respectively.

Now, $V_{\text {Max }}=\operatorname{Max}\left(V_{1}, V_{2}, V_{3}, V_{4}\right)=\operatorname{Max}(1,0.831,0.96,0.85)=1$. From the theory, we know that, $W_{i}=\left\{V_{i} / \operatorname{Max}\left(V_{1}, V_{2}, V_{3}, V_{4}\right)\right\}^{2}$. Therefore, $W_{1}=(1 / 1)^{2}=1, W_{2}=(0.831 / 1)^{2}=0.69, W_{3}=(0.96 / 1)^{2}=0.92$, and $W_{4}=(0.85 / 1)^{2}=0.722$

\subsubsection{Numerical example-2}

We take test data as $\left(\mathrm{SU}_{1}, \mathrm{SU}_{2}, \mathrm{SU}_{3}, \mathrm{SU}_{4}\right) \equiv\{(0.92,0.51,1.61,1.72), 1\}$. From the membership function of SL,

$\Psi_{01}\left(\mathrm{SU}_{1}=0.92\right)=0.62 \leftrightarrow B \in\{A, B\}$ i.e., $B$ belongs to the first set of $R_{0}$ $\Psi_{02}\left(\mathrm{SU}_{2}=0.51\right)=0.91 \leftrightarrow A \in\{A, B\}$ i.e., $A$ belongs to the second set of $R_{0}$ $\Psi_{03}\left(\mathrm{SU}_{3}=1.61\right)=0.74 \leftrightarrow B \in\{A, B\}$ i.e., $B$ belong to the third set of $R_{0}$ $\Psi_{04}\left(\mathrm{SU}_{4}=1.72\right)=0.78 \leftrightarrow B \in\{A, B\}$ i.e., $B$ belong to the fourth set of $R_{0}$ The weighted co-variance of Fuzzy rule $R_{0}$,

$$
R=\sum_{i=1}^{4} \Psi_{0 i}\left(X_{i}\right) W_{i}=1 * 0.62+0.69 * 0.91+0.92 * 0.74+0.722 * 0.78=2.49
$$

$\Psi_{11}\left(\mathrm{SU}_{1}=0.92\right)=0.62 \leftrightarrow B \notin\{C, D\}$ i.e., $B$ does not belong to the first set of $R_{1}$ $\Psi_{12}\left(\mathrm{SU}_{2}=0.51\right)=0.91 \leftrightarrow A \notin\{B, C\}$ i.e., A does not belong to the second set of $R_{1}$ $\Psi_{13}\left(\mathrm{SU}_{3}=1.61\right)=0.74 \leftrightarrow B \in\{\mathrm{B}, \mathrm{C}, \mathrm{D}\}$ i.e., $\mathrm{B}$ belong to the third set of $R_{1}$ $\Psi_{14}\left(\mathrm{SU}_{4}=1.72\right)=0.78 \leftrightarrow B \in\{C, B, D, E\}$ i.e., B belong to the fourth set of $R_{1}$ The weighted co-variance of Fuzzy rule $R_{1}$,

$$
R=\sum_{i=1}^{4} \Psi_{1 i}\left(X_{i}\right) W_{i}=0+0+0.92 * 0.74+0.722 * 0.78=1.24
$$

The maximum value of $R$ is found for rule $R_{0}$; therefore, $(0.92,0.51,1.61,1.72)$ supports $R_{0}$ i.e., the testing data is under hypothesis $H_{0}$, which is found to be correct.

\subsection{Fuzzy inference system}

Fuzzy Inference System (FIS) relates input vectors $\mathbf{X}=\left[C_{0} C_{1} C_{2} \ldots C_{k}\right]$, each of size $k$, to output variable $Y$ using Fuzzy logic. A FIS consists of three blocks named Fuzzification block, Inference engine and De-fuzzifier block as explained in [18-21] for different applications. In this paper, we use the following steps to relate the signals of SUs at FC with the decision of hypothesis $H_{0}$ or $H_{1}$.

a) Take M samples from the signal $s(t)$ of each of SUs at FC.

b) Apply recurrent discrete wavelet transform on the sample vector until reducing it to a size of 4 as $\mathbf{V}=$ $\left[\mathrm{C}_{0} \mathrm{C}_{1} \mathrm{C}_{2} \mathrm{C}_{3}\right]$

c) Apply vectors $\mathbf{V}$ to FIS

d) Generate crisp output $\mathrm{Y}$ as 0 or 1 against the hypothesis $\mathrm{H}_{0}$ or $\mathrm{H}_{1}$

The result section reveals the signal vector $\mathbf{V}$ and corresponding output $Y$ in a tabular form.

\subsection{Fuzzy $c$-means clustering}

Here, data is separated into several clusters, which may be overlapping or non-overlapping. The distance between the center of a cluster and the point under consideration governs the grade of a MF. The shorter the distance, the higher the grade of a MF. The steps of Fuzzy $c$-Means Clustering algorithm is available in [22-24]. In this paper, we take the received signal of PUs at FC under three categories: Hypothesis $H_{0}$ (absence of PU), Hypothesis $H_{1}$ (presence of PU) and Hypothesis $H_{0}^{+}$(intermediate result, usually applicable to malicious attack); where SUs are used as the relay stations. Next, we apply Fuzzy $c$ Means Clustering algorithm to get the scatterplot of data after convergence of three degree of belongings: $\mathbf{U}_{1}(k), \mathbf{U}_{2}(k)$ and $\mathbf{U}_{3}(k)$ of three hypotheses. 


\subsection{Support vector machine}

A Support Vector Machine (SVM) is a machine learning model for the classification of response data of a system. The basic concept of SVM is to construct a linear or non-linear hyperplane to separate the data points under different conditions. As an example, let us consider a set of data $\left\{x_{i}\right\}, i=0,1,2, \ldots,(N-1)$, and corresponding desired response of a system is, $d_{i} \in\{+1,-1\}$, which is represented as the set of ordered pair, $\left\{\left(x_{i}, d_{i}\right)\right\}_{i=0}^{N-1}$. The equation of hyperplane, $\mathbf{w}^{\mathrm{T}} \mathbf{x}+\mathrm{b}=0$ (where $\mathbf{x}$ is input vector, $\mathbf{w}$ is weight vector and $b$ is a bias) satisfies, $\mathbf{w}^{\mathrm{T}} x_{i}+\mathrm{b} \geq 0$ for $d_{i}=+1$ and $\mathbf{w}^{\mathrm{T}} x_{i}+\mathrm{b}<0$ for $d_{i}=-1$. Higher degree polynomial or even a special function like Gaussian Radial Basis Function is used as a hyperplane to segregate complex data [10-11]. We also consider three types of data under hypothesis $H_{0}$, hypothesis $H_{1}$ and hypothesis $H_{0}{ }^{+}$. Here, the input vector is SINR at FC and we determine SINR at receiving end as a random variable using the concept of [25$26]$.

\subsection{Convolutional neural network}

A Convolutional Neural Ntwork $(\mathrm{CNN})$ is one kind of Deep Neural Network (DNN) that acquires an immense popularity in object recognition. The main functional block of a CNN is convolutional layer in which a Linear Time Invariant (LTI) system is activated as $y(t)=x(t)^{*} h(t)$; where $x(t)$ is input signal, $h(t)$ is impulse response of LTI system and $y(t)$ is output of the system. If LTI system is a filter, then the convolutional operation provides filtered signal. In CNN, we use the term "convolutional filter" or "kernel" against the impulse response $h(t)$ and feature map for output signal $y(t)$.

Each convolutional layer is followed by a pooling layer and we consider an average pooling technique. Next, the Rectified Linear Unit (ReLU) works as an activation function like the threshold of signal. The output of the ReLU is connected to a fully connected NN to produce feature corresponding to hypothesis $H_{0}$ and $H_{1}$ as shown in Figure 3. The received signal at FC from several SUs are converted into an image. The noisy image is applied to CNN to take the decision about the presence or absence of a PU taking the expression as shown in (4) and (8) of SINR of single user and multiuser model of [27-28].
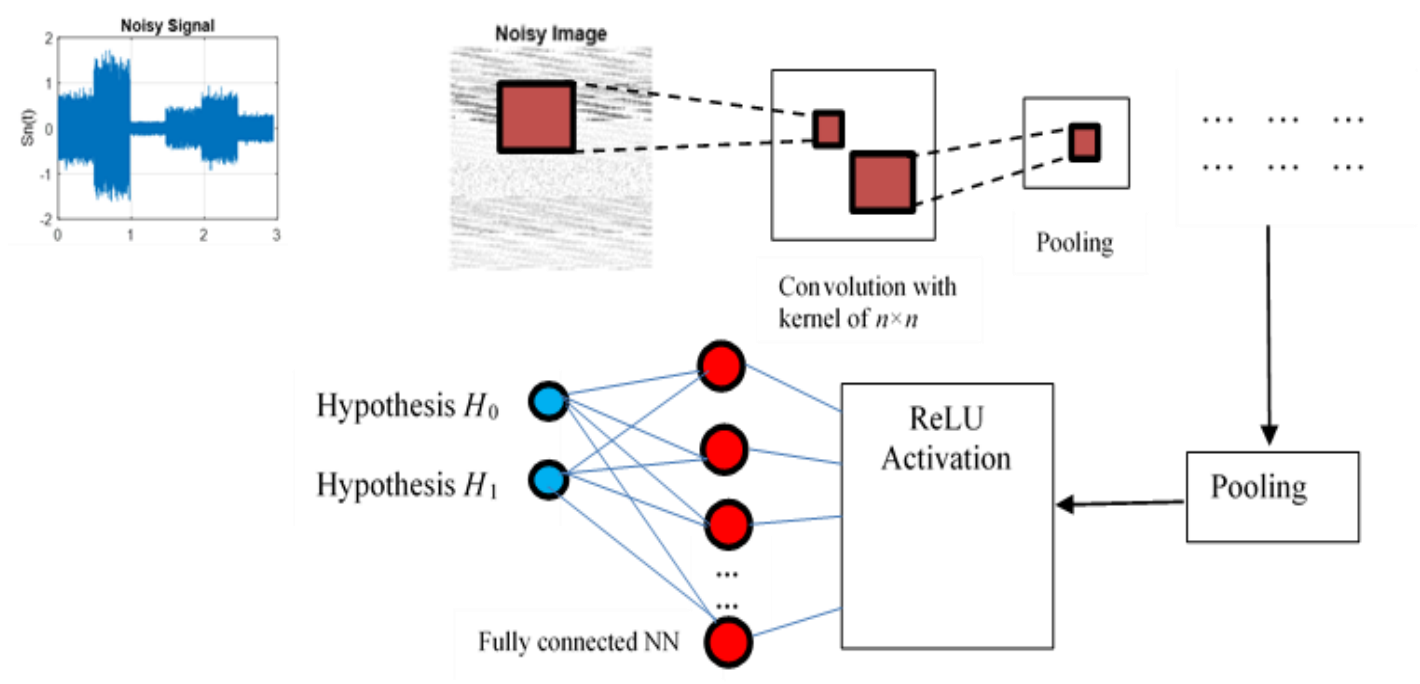

Figure 3. Basic building block of $\mathrm{CNN}$ to recognize signal at FC

\subsubsection{Simulation algorithm}

a) Set the link parameters as mention in result section and $\varepsilon=2$

b) Assign the transmitted power, $\mathrm{P}=\operatorname{rand}() ; \%$ average power of 0.5 under $\mathrm{H}_{0}$

c) $\mathrm{N}=49 ; \%$ size of image is $49 \times 49$

for $\mathrm{i}=1: \mathrm{N}$

for $\mathrm{j}=1: \mathrm{N}$

Store SINR for multi user as, Gamma_m(i,j) using eq. (8) of [27]

Store SINR for single user as, Gamma_s(i,j) using eq. (4), of [27] as mentioned before end end 
d) Repeat step c taking, $\mathrm{P}=\operatorname{rand}()+5 ; \%$ average Power of 5 under $\mathrm{H}_{1}$

e) Repeat step a to $\mathrm{d}$ for $\varepsilon=2.25$ and 2.5

f) Create image for matrices Gamma_s and Gamma_m

g) Store 10 images for each category in a folder

h) Apply the image to a CNN taking appropriate parameter of NN.

i) Acquire the features of the image and take decision about hypothesis $\mathrm{H}_{0}$ or $\mathrm{H}_{1}$

\section{RESULTS AND DISCUSSION}

First, we concentrate on the results of Fuzzy weighted rule. However, our prime focus is on the results of four machine learning techniques. Here, we consider four SUs as relay station under a FC. Only a few received data under hypothesis $H_{1}$ and $H_{0}$ are shown in Table 1 . About100 data sets representing the received signal under a Rayleigh fading channel along with AWGN like [29] are taken for simulation. Working on 12 data set (each data set contains 100 records like Table 1), we get the outcome of Fuzzy weighted rule for five different experiments on simulated signal as shown in Table 3.

The next part of the experiment deals with FIS. The signal vectors correspoding to section 2.2 are shown in Table 4 for both $H_{0}$ and $H_{1}$ using 16-QAM signal with AWGN and Rayleigh fading of [30] at FC, and simulation is done 500 times for each hypothesis and only 9 of them are shown. The verification of Fuzzy rules is carried out against $H_{0}$ and $H_{1}$ with three numerical values for vector $\mathbf{V}$ as $\mathbf{V}_{1}=\left[\begin{array}{ll}1 & 0.0198\end{array}\right.$

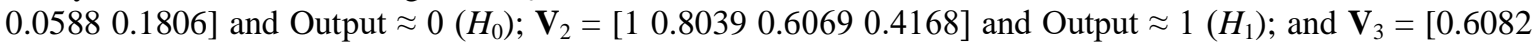
$0.198910 .3649]$ and Output $\approx 0\left(H_{0}\right)$, respectively.

Table 3. Signal detection with Fuzzy weighted rule

\begin{tabular}{ccccc}
\hline Experiment No. & $\begin{array}{c}\text { Detection of } H_{0} \\
\text { (2 SUs at FC) }\end{array}$ & $\begin{array}{c}\text { Detection of } H_{1} \\
\text { (2 SUs at FC) }\end{array}$ & $\begin{array}{c}\text { Detection of } H_{0} \\
\text { (4 SUs at FC) }\end{array}$ & $\begin{array}{c}\text { Detection of } H_{1} \\
\text { (4 SUs at FC) }\end{array}$ \\
\hline 1 & 0.832 & 0.858 & 0.873 & 0.892 \\
2 & 0.803 & 0.869 & 0.886 & 0.883 \\
3 & 0.838 & 0.876 & 0.865 & 0.874 \\
4 & 0.847 & 0.811 & 0.869 & 0.867 \\
5 & 0.823 & 0.832 & 0.847 & 0.881 \\
\hline
\end{tabular}

Table 4. Signal vectors for FIS

\begin{tabular}{cccccccccc}
\hline$C_{0}$ & $C_{1}$ & $C_{2}$ & $C_{3}$ & $H_{1}$ & $C_{0}$ & $C_{1}$ & $C_{2}$ & $C_{3}$ & $H_{0}$ \\
\hline 0.1500 & 0.1445 & 1.0000 & 0.0426 & 1 & 1.0000 & 0.0198 & 0.0588 & 0.1806 & 0 \\
0.2140 & 0.8811 & 0.6402 & 1.0000 & 1 & 1.0000 & 0.0233 & 0.1348 & 0.1856 & 0 \\
1.0000 & 0.0148 & 0.2177 & 0.0845 & 1 & 0.9467 & 0.2660 & 0.0756 & 1.0000 & 0 \\
0.2458 & 0.1493 & 1.0000 & 0.5703 & 1 & 1.0000 & 0.0881 & 0.0381 & 0.4125 & 0 \\
1.0000 & 0.8039 & 0.6069 & 0.4168 & 1 & 1.0000 & 0.0684 & 0.4053 & 0.1556 & 0 \\
1.0000 & 0.0571 & 0.2505 & 0.2533 & 1 & 0.6082 & 0.1989 & 1.0000 & 0.3649 & 0 \\
0.1565 & 0.3330 & 0.4324 & 1.0000 & 1 & 1.0000 & 0.0293 & 0.6662 & 0.0993 & 0 \\
1.0000 & 0.3667 & 0.1601 & 0.1698 & 1 & 0.9793 & 0.0692 & 0.0430 & 1.0000 & 0 \\
1.0000 & 0.0111 & 0.3373 & 0.1485 & 1 & 0.6517 & 0.5511 & 0.7855 & 1.0000 & 0 \\
\hline
\end{tabular}

Now, the experiment deals with Fuzzy $c$-Means Clustering (FCMC). The scatterplot of data set of $H_{0}, H_{1}$ and $H_{0}+$ under FCMC is shown in Figure 4. After 61 iterations, we get three distinct regions on scatterplot; where the function $U(\mathrm{k})$ takes the numerical values of $U(56)=594.730209, U(57)=594.730207$, $U(58)=594.730205, U(59)=594.730204, U(60)=594.730203, U(61)=594.730202$, which are very close. We run simulation 50 times in Matlab v.18 and get the detection accuracy of $78.246 \%$ as the best case and of $73.215 \%$ as the worst case. If we use two hypothesis model i.e., excluding the data set of intermediate level $\mathrm{H}_{0}{ }^{+}$, then we get the detection accuracy of $94.113 \%$ as the best case and of $88.512 \%$ as the worst case.

Next, we apply SVM on the simulated random data of SINR and the corresponding scatterplot is shown in Figure 5(a) and the region of $H_{0}, H_{1}$ and $\mathrm{H}_{0}{ }^{+}$is shown in Figure 5(b). The SVM seems to be more successful approach than that of FCMC. The success rate for 200 random data is of $96.234 \%$ as the best case and of $92.678 \%$ as the worst case.

Finally, we apply CNN on received signal under Rayleigh fading and AWGN channel captured at FC. We consider 16-QAM signal and the duration of six consecutive symbols as time slot. The fading signal of length 4900 (one time slot) is converted to an image of $49 \times 49$ using the algorithm of section 2.5.1. The signal of a time slot and the corresponding images are shown in Figure 6(a) and 6(b) under hypothesis $H_{1}$ and $H_{0}$, respectively. We make 100 images for each category, and then apply deep learning algorithm e.g., CNN. Running CNN several times, we measure the accuracy of detection for three cases as shown in Figure 7.

Improving signal detection accuracy at FC of a CRN using machine learning ... (Md Abul Kalam Azad) 

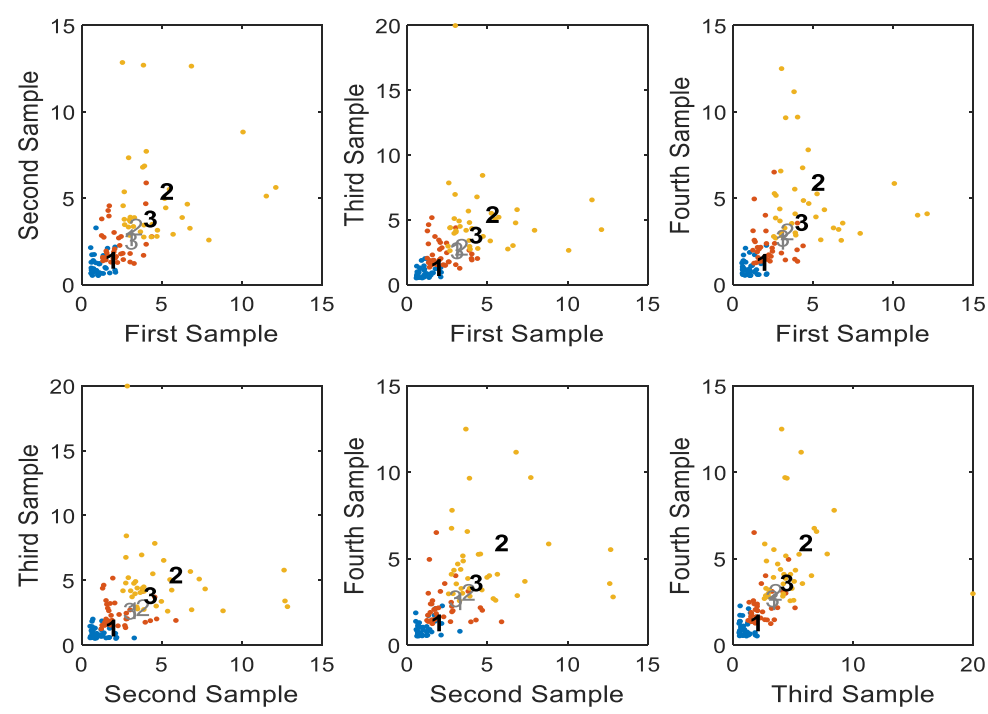

Figure 4. Scatterplot of Fuzzy c-mean clustering with three distinct region

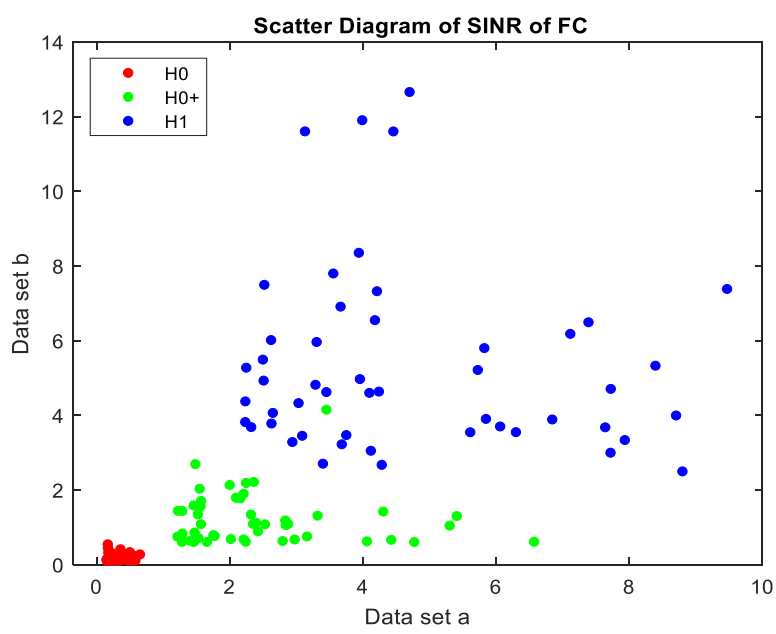

(a) Scatter plot of data set

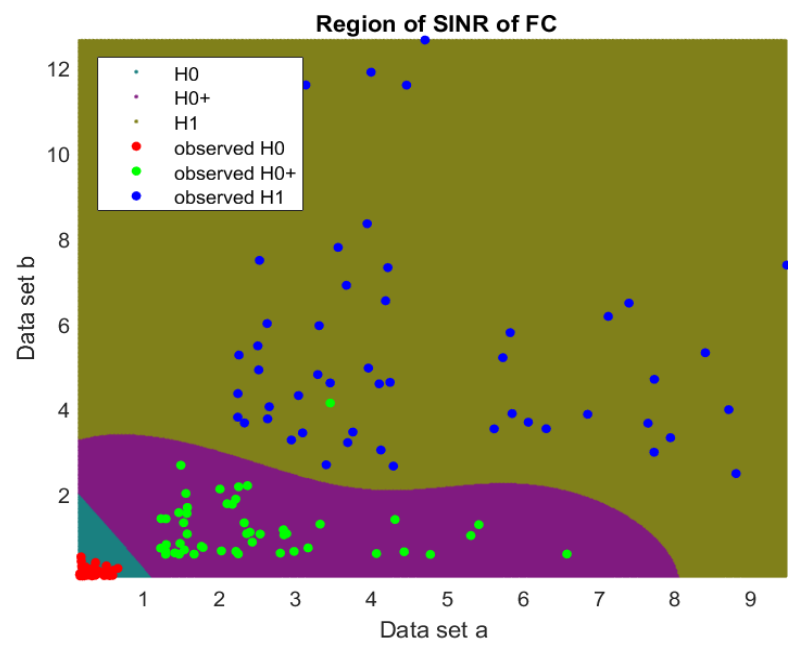

(b) Region using SVM

Figure 5. Scatterplot of two hypothesis model under SVM 

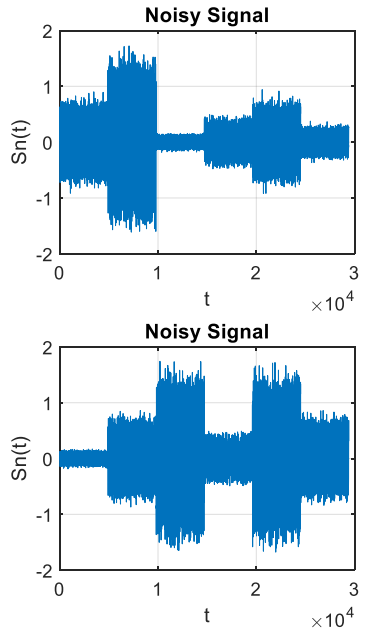

(a) Noisy signal and image under $H_{1}$
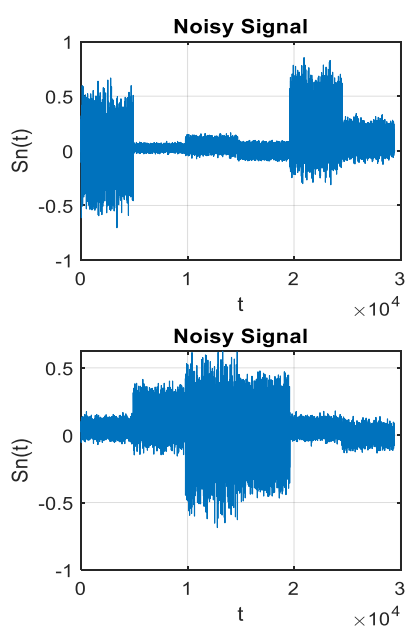

(b) Noisy signal and image under $H_{0}$
Noisy Image

Noisy Image

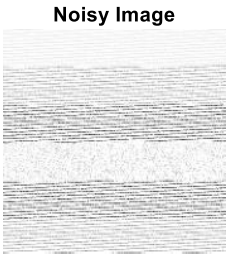

Figure 6. 16-QAM signal and corresponding image at FC

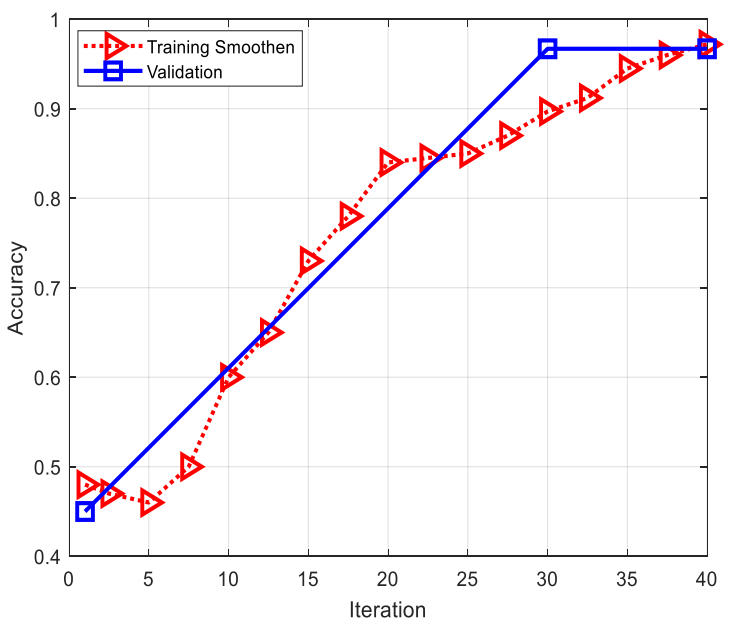

(a) Worst case

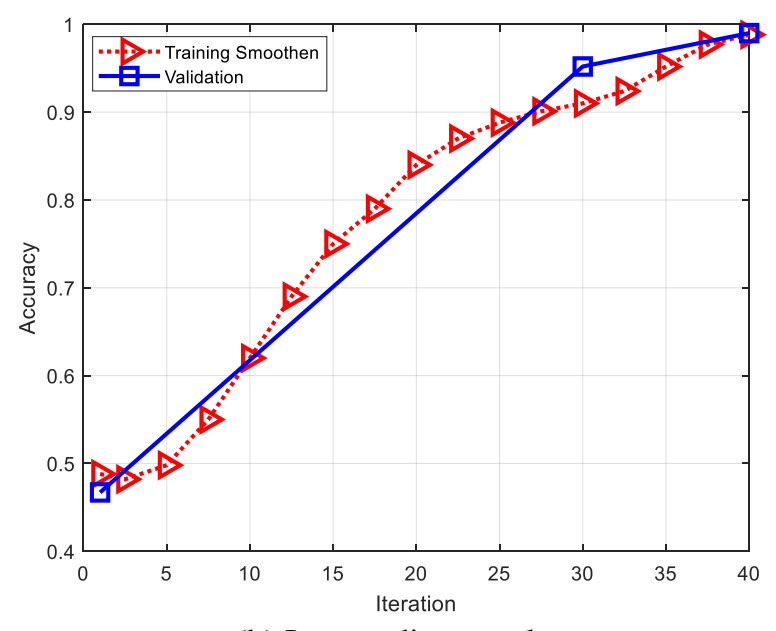

(b) Intermediate result

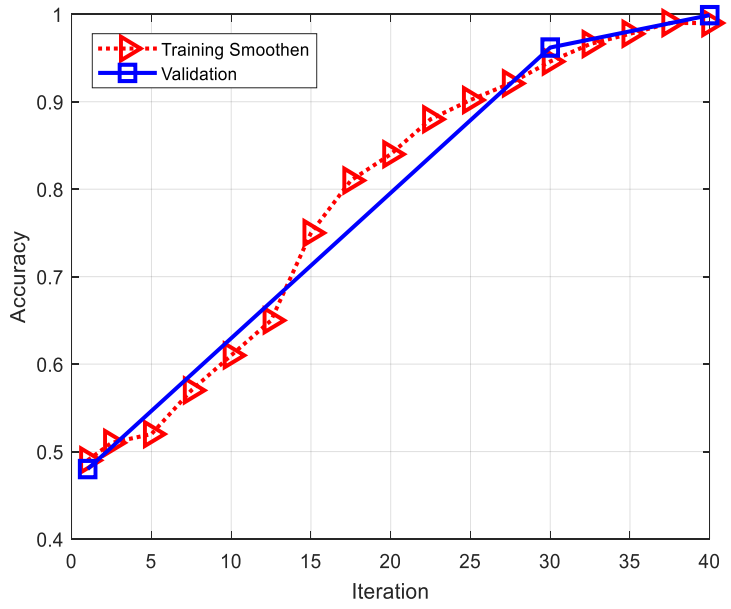

(c) Best case

Figure 7. Accuracy of detection from $\mathrm{CNN}$ 
Finally, the outcomes of five different methods are combined together to achieve a decision about the presence or absence of a PU in CRN. To combine five methods, we use the following algorithm based on the idea of [29]:

a) If the accuracy of recognition of $i$ th method (for example SVM) is $a_{i}$ then the accuracy vector of 5 methods is $\mathbf{V}_{a}=\left[a_{1}, a_{2}, a_{3}, a_{4}, a_{5}\right]$.

b) Normalize the accuracy vector as, $\mathbf{V}_{n}=\left[a_{1}, a_{2}, a_{3}, a_{4}, a_{5}\right] / \sum_{i=1}^{5} a_{i}=\left[b_{1}, b_{2}, b_{3}, b_{4}, b_{5}\right]$

c) Determine the entropy of elements of $\mathbf{V}_{n}, E=\sum_{i=1}^{5} b_{i} \log _{2}\left(\frac{1}{b_{i}}\right)$, which has the maximum value of 2.3219 .

d) If $E>2.2$ and majority of the methods ( 3 out of five) has $a_{i}>0.75$, we consider the detection is correct.

e) Repeat all steps $\mathrm{M}$ time and determine the ratio of correct decision and $\mathrm{M}$, which the accuracy of combined method.

f) The correct decision about $\mathrm{H}_{0}$ and $\mathrm{H}_{1}$ are averaged.

The combined result of above algorithm is shown in Table 5, where we found that the combined method gives a better result than that of any individual classification technique.

Table 5. Comparison of experimental results

\begin{tabular}{ccccccc}
\hline Experiment Number & Weighted Fuzzy System & FIS & Fuzzy $c$-Means Clustering & SVM & CNN & Combined \\
\hline 1 & 0.836 & 0.873 & 0.782 & 0.763 & 0.894 & 0.962 \\
2 & 0.811 & 0.849 & 0.765 & 0.724 & 0.873 & 0.958 \\
3 & 0.829 & 0.881 & 0.791 & 0.783 & 0.901 & 0.967 \\
4 & 0.802 & 0.847 & 0.752 & 0.772 & 0.843 & 0.925 \\
5 & 0.866 & 0.891 & 0.787 & 0.782 & 0.913 & 0.971 \\
\hline
\end{tabular}

\section{CONCLUSION}

In this paper, Fuzzy system and four different machine learning techniques are used at FC to detect the presence or absence of a PU. Here, CNN shows the best result among all classification techniques whereas SVM shows the worst. However, the combined method gives the best classification outcome with an accuracy of detection about $96.7 \%$. Still, we have the scope to observe the performance of other machine learning algorithms such as Principal Component Analysis, Linear Discriminant Analysis, Speeded-Up Robust Features, Scale-Invariant Feature Transform, etc. In future, we will include malicious user attack into CRN using three hypothesis model under different machine learning algorithms.

\section{REFERENCES}

[1] Y. -C. Chen, et al., "Generating weighted fuzzy rules from training data for dealing with the iris data classification problem," International Journal of Apllied Science and Engineering, vol. 4, pp. 41-52, 2006.

[2] R. A. Mohammadpour, et al., "Fuzzy rule-based classification system for assessing coronary artery disease," Computational and Mathematical Methods in Medicine, vol. 2015, pp. 1-8, 2015.

[3] R. M. Prakash and R. S. S. Kumari, "Fuzzy C means integrated with spatial information and contrast enhancement for segmentation of MR brain images," International Journal of Imaging Systems and Technology, vol. 26, pp. 116123, 2016.

[4] P. O. Gokten, et al., "Using fuzzy c-means clustering algorithm in financial health scoring," The Audit Financiar Journal, vol. 15, pp. 385-394, 2017.

[5] C. Li, et al., "Image segmentation based on fuzzy clustering with cellular automata and features weighting," EURASIP Journal on Image and Video Processing, pp. 1-11, 2019.

[6] D. A. Reyes, et al., "Classifying hand movement intentions using surface EMG signals and SVM," in 2019 XXII Symposium on Image, Signal Processing and Artificial Vision (STSIVA), pp. 24-26, 2019.

[7] M. S. Refahi, et al., "ECG arrhythmia classification using least squares twin support vector machines," in 26th Iranian Conference on Electrical Engineering (ICEE2018), pp. 1619-1621, 2018.

[8] I. B. Aydilek, "Examining effects of the support vector machines kernel types on biomedical data classification," in 2018 International Conference on Artificial Intelligence and Data Processing (IDAP), pp. 1-4, 2018.

[9] Z. dandan and Z. Xuping, "SVM-based spectrum sensing in cognitive radio," in 2011 7th International Conference on Wireless Communications, Networking and Mobile Computing, pp. 1-4, 2011.

[10] Y. -D. Huang, et al., "A Fuzzy support vector machine algorithm for cooperative spectrum sensing with noise uncertainty," in 2016 IEEE Global Communications Conference (GLOBECOM), pp. 1-6, 2016.

[11] O. P. Awe, et al., "Eigen value and support vector machine techniques for spectrum sensing in cognitive radio networks," in 2013 Conference on Technologies and Applications of Artificial Intelligence, pp. 223-227, 2013. 
[12] Y. Lu, et al., "Machine learning techniques with probability vector for cooperative spectrum sensing in cognitive radio networks," in IEEE Wireless Conference and Networking Conference (WCNC 2016), pp.1-6, 2016.

[13] K. M. Thilina, et al., "Machine learning techniques for cooperative spectrum sensing in cognitive radio networks," IEEE Journal on Selected Areas in Communications, vol. 31, no. 11, pp. 2209-2221, 2013.

[14] M. Ueda, et al., "An age estimation method using 3d-CNN from brain MRI images," in 2019 IEEE 16th International Symposium on Biomedical Imaging (ISBI 2019), pp. 380-383, 2019.

[15] K. Jaiswal and D. K. Patel, "Sound classification using convolutional neural networks," in 2018 IEEE International Conference on Cloud Computing in Emerging Markets (CCEM), pp. 81-84, 2018.

[16] P. Yang, et al., "Dynamic spectrum access in cognitive radio networks using deep reinforcement learning and evolutionary game," in 2018 IEEE/CIC International Conference on Communications in China (ICCC), pp. 405409, 2018.

[17] H. Liu, et al., "Ensemble deep learning based cooperative spectrum sensing with semi-soft stacking fusion center," in 2019 IEEE Wireless Communications and Networking Conference (WCNC), pp. 1-6, 2019.

[18] S. M. Taheri, et al., "Application of Fuzzy inference systems in archaeology," in 2019 7th Iranian Joint Congress on Fuzzy and Intelligent Systems (CFIS), pp. 1-4, 2019.

[19] M. Alrashoud, "Hierarchical fuzzy inference system for diagnosing dengue disease," in 2019 IEEE International Conference on Multimedia \& Expo Workshops (ICMEW), pp. 31-36, 2019.

[20] K. Kuspijani, et al., "Fault classification of induction motor using discrete wavelet transform and Fuzzy inference system," in 2020 International Conference on Smart Technology and Applications (ICoSTA), pp. 1-6, 2010.

[21] M. Mazandarani and X. Li, "Fractional Fuzzy inference system: The new generation of Fuzzy inference systems," IEEE Access, vol. 8, pp. 126066-126082, 2020.

[22] M. B. Panna and M. I. Islam, "human face detection based on combination of linear regression, PCA and Fuzzy cmeans clustering," International Journal of Computer Science and Information Security, vol. 17, no. 7, pp. 57-62, 2019.

[23] X. Zhang, et al., "Robust image segmentation using Fuzzy c-means clustering with spatial information based on total generalized variation," IEEE Access, vol. 8, pp. 95681-95697, 2020.

[24] P. Bo, et al., "A cloud and cloud shadow detection method based on Fuzzy c-means algorithm," IEEE Journal of Selected Topics in Applied Earth Observations and Remote Sensing, vol. 13, pp. 1714-1727, 2020.

[25] M. Xia and S. Aïssa, "Modeling and Analysis of Cooperative Relaying in Spectrum-Sharing Cellular Systems," IEEE Transactions on Vehicular Technology, vol. 65, no. 11, pp. 9112-9122, 2016.

[26] S. Y. Chaganti, et al., "Image classification using SVM and CNN," in 2020 International Conference on Computer Science, Engineering and Applications (ICCSEA), pp. 1-5, 2020.

[27] M. A. K. Azad, et al., "Comparison of performance of cognitive radio network under single and multi-user scenario," in 2019 1st International Conference on Advances in Science, Engineering and Robotics Technology (ICASERT 2019), pp. 1-5, 2019.

[28] B. P. Amiruddin and R. E. A. Kadir, "CNN architectures performance evaluation for image classification of mosquito in indonesia," in 2020 International Seminar on Intelligent Technology and Its Applications (ISITIA), pp. 223-227, 2020.

[29] F. Tabassum, et al., "Human face recognition with combination of DWT and machine learning," Journal of King Saud University - Computer and Information Sciences (Elsevier), Feb. 2020. doi: 10.1016/j.jksuci.2020.02.002.

[30] A. K. Azad, et al., "Signal detection of Co-operative cognitive radio network under neural network," Journal of Computer and Communications, vol. 6, no. 9, pp. 60-72, 2018.

\section{BIOGRAPHIES OF AUTHORS}

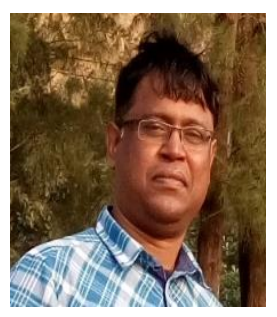

Md. Abul Kalam Azad has completed his Bachelor of Science with Honors in Electronics and Computer Science from Jahangirnagar University, Dhaka, Bangladesh and Master of Science in Information Technology from Royal Institute of Technology (KTH), Sweden. Currently, Mr. Azad is working as a Professor in the department of Computer Science \& Engineering, Jahangirnagar University, Dhaka, Bangladesh. His research interest includes wireless networks, particularly in wireless sensor networks, Ad-Hoc networks, and mobile cognitive networks.

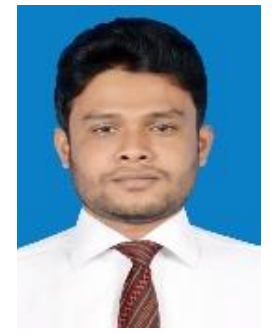

Anup Majumder received his B.Sc. (Honors) and M.Sc. in Computer Science and Engineering from Jahangirnagar University, Dhaka, Bangladesh in 2014 and 2015 respectively. Previously, he worked as a lecturer in the Department of Computer Science and Engineering, Daffodil International University, Dhaka, Bangladesh and also worked as a lecturer at the Institute of Information Technology, Noakhali Science and Technology University, Noakhali, Bangladesh. Currently, he is working as a lecturer in the Department of Computer Science and Engineering, Jahanginagar University, Dhaka, Bangladesh. His research interest is focused on Machine Learning and Expert System, Data Mining and wireless network. 


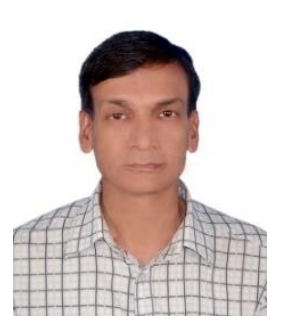

Jugal Krishna Das has completed his Ph. D. from Glushkov Institute of Cybernetics, Kiev, Ukraine, in 1993 and M. Sc. from Donetsk Polytechnic Institute, Ukraine, in 1989. Now he is working as a Professor in the department of Computer Science and Engineering, Jahangirnagar University, Savar, Dhaka, Bangladesh. He is the author of 13 Journal and more than 16 International Conference papers in home and abroad. His research interests include Network Protocols, Universal Networking Language, Distributed Systems, and so on.

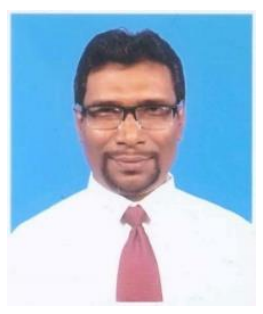

Md. Imdadul Islam completed his B.Sc. and M.Sc Engineering in EEE from BUET, Dhaka, Bangladesh in 1993 and 1998, respectively and completed his PhD from the Department of CSE, Jahangirnagar University, Dhaka, Bangladesh in 2010. He is now working as a Professor at the Department of CSE, Jahangirnagar University, Dhaka, Bangladesh. Previously, he worked as an Assistant Engineer in Sheba Telecom (Pvt.) LTD (A joint venture between Bangladesh and Malaysia for Mobile cellular and WLL) from Sept.1994 to July 1996. Dr. Islam has a very good field experience in installation and design of mobile cellular network, Radio Base Stations and Switching Centers for both mobile and WLL. His research fields are network traffic, wireless communications, wavelet transform, OFDMA, WCDMA, adaptive filter theory, array antenna systems, ANFIS and machine learning. He published more than hundred and eighty research papers in national and international journals and conference proceedings. 